\title{
Edaravone's Antioxidant Capabilities and its Therapeutic Benefits for Post-Ischemic Stroke: A Mini Review
}

\author{
Hunter Rolain, Gurwattan S Miranpuri and Azam S Ahmed* \\ Department of Neurological Surgery, University of Wisconsin School of Medicine and Public Health, USA
}

*Corresponding author: Azam S Ahmed, Neurosurgeon, University of Wisconsin School of Medicine and Public Health, Madison, USA.

Received Date: September 26, 2019

Published Date: October 30, 2019

\begin{abstract}
Acute-ischemic stroke (AIS) is one of the leading causes of death worldwide. IV tPA and thrombectomy have been effective in recanalizing occluded vessels. However, there is often a delay in the thrombolytic effect or completing the thrombectomy. Neuroprotective agents may bridge the gap and slow the conversion of ischemic to infarcted brain. Edaravone is a potent antioxidant capable of sequestering free radicals, reactive oxygen species (ROS) and inhibiting oxidative stress. This mini review highlights edaravone as potential antioxidant, thrombolytic adjunct, and prophylactic for cerebral edema.
\end{abstract}

Keywords: Edaravone, Tissue plasminogen activator, tPA, cerebral infarction, Amyotrophic lateral sclerosis, ALS, acute-ischemic stroke, AIS, ROS, antioxidants, neurodegenerative diseases, oxidative stress

\section{Introduction}

A stroke occurs every 40 seconds with someone dying every four minutes [1]. It is one of the leading causes of death not only in the US but also worldwide [2]. Worldwide, among the patients who survive most remain permanently disabled, thereby compounding the financial impact to society in lost productivity and cost for care [1].

One of the main pathways leading to cell death in ischemic stroke is oxidative stress. Free radical toxicity contributes to an array of cellular damage including inflammation, protein degradation, DNA damage and cellular apoptosis [3]. When combined, these processes all lead to tissue damage and ultimately cell death [3]. Due to a variety of factors, the tolerance to ischemia differs based on collateral circulation pathways, genetic and epigenetic differences $[4,5]$. Regardless of these individual differences, infractions extend with time ultimately resulting in irreversible injury to a vascular territory.

In attempts to halt the conversion of ischemic to infarcted brain, thrombolytic therapy along with endovascular thrombectomy have been used to lyse the clot or mechanically remove it. However, only
3 percent of patients actually arrive at a time window indicated for the administration of IV tPA (less than 4.5 hours) [6]. Patients with large vessel occlusions with a perfusion pattern indicative of salvageable ischemic brain are now treated with endovascular thrombectomy [7]. With either treatment, and particularly for endovascular treatment, time is required to perform the procedure or if diagnosed at non-thrombectomy capable centre, arrange for transfer to a comprehensive stroke centre $[8,9]$.

Neuronal loss continues until reperfusion is achieved at a rate of 1.9 million each minute, on average [10]. Even once reperfusion is achieved, "reperfusion injury" to ischemic brain may occur $[11,12]$. Neuroprotective agents may temporize the situation thereby slowing the conversion rate of ischemic to infarcted brain prior to recanalization or to limit reperfusion injury [13-15].

Edaravone, a free radical scavenger improves functional outcomes through free radical scavenging and potentially enhanced thrombolysis when used as an adjunct to IV tPA.16 Since completion of a pivotal trial in 2001, edaravone has been used routinely in Japan to treat patients for acute-ischemic stroke $[6,16,17]$. 
Herein, we discuss the mechanisms of action, safety, and efficacy of edaravone in the acute stroke patient populations. We also identify future directions of study for this promising therapy.

\section{Edaravone: A Potent Antioxidant}

Ischemic stroke results in oxidative stress and the generation of harmful free radicals and reactive oxygen species (ROS) [3]. These lead to inflammation, tissue damage, and cellular apoptosis by attacking cell membranes and the peroxidation of unsaturated fatty acids into phospholipids $[3,18]$. The c-Jun N-terminal kinase (JNK) pathway is known to play an influential role in inflammation, cytokine production and cellular apoptosis triggered by oxidative stress [19]. ROS results in the phosphorylation of JNK and leads to multiple death factors $[19,20]$. Through recent research edaravone is thought to target the JNK pathway and inhibit this "death" pathway
[21]. Edaravone limits free radical and ROS due to its conjugated aromatic system. Furthermore, antioxidants like edaravone have the capability to sequester metal ions to prevent oxidative stress for pre-stroke patients [3]. In many studies the amphiphilic edaravone was found to have a positive correlation with ROS and free radical reduction as well as reduced infarct volume and neurological deficits $[3,18,22,23]$. Secondary injurious processes (e.g cerebral edema, inflammation) are limited by free-radical and ROS reduction. Secondary to the reduction of ROS, edaravone even has the capability to reduce the production of inflammatory cytokines, cytokine-induced neutrophil chemoattractant, and nitric oxide synthases [3]. Thus, upstream and downstream antioxidant therapy may be a beneficial treatment in altering neuronal damage, limiting edema and recovering the penumbra [3].

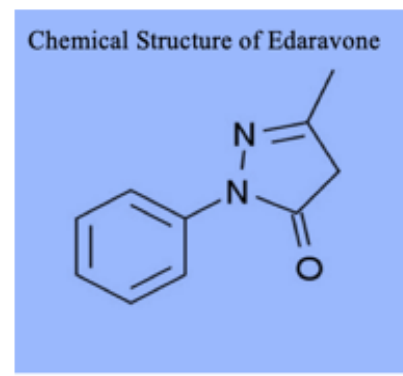

\section{Ischemia/Stress}

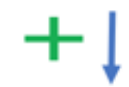

Oxidative Stress

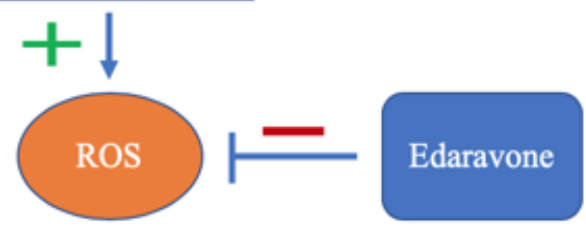

$+\mid$ MKK $4 / \mathrm{MKK} 7$

JNK
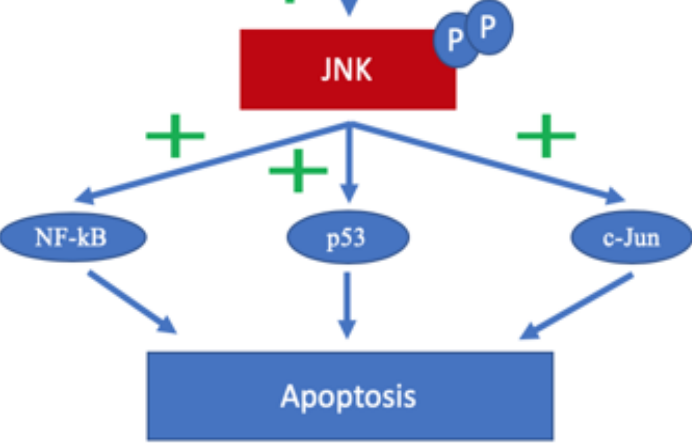

Figure 1: Edaravone chemical structure and possible mechanism of JNK pathway.

\section{Safety}

\section{Intracerebral haemorrhage}

Ischemic stroke leads to blood brain barrier disruption and may result in intracerebral haemorrhage. The greater the volume of infarcted brain, the more likely haemorrhagic transformation is to occur. While it is obvious IV tPA may increase the likelihood of haemorrhagic transformation due to its direct thrombolytic effect. Recently increased MMP-9 concentrations, linked to haemorrhagic transformation in ischemic stroke, have been linked to IV tPA administration [12,24,25]. Oxidative stress, BBB disruption and mitochondrial mechanisms are all possibly involved in this pathophysiology that lead to haemorrhagic transformation and increased infarct growth $[12,24,25]$. It is therefore imperative that neuroprotective agents not increase this risk further.

Edaravone causes an increase vasodilation through the production of prostacyclin's and nitric oxide leading to the theoretical possibility of an even greater increase in ICH $[6,17,26,27]$. However, when $30 \mathrm{mg}$ edaravone was administered along with IV tPA to patients presenting with M1 or M2 segment of the middle cerebral artery occlusions within 4.5 hours, whether before, during or after IV tPA administration, ICH only occurred in 4 patients $(2 \%)$ and 2 patients $(2 \%)$ in the early and late groups, respectively[28]. This difference was not statistically significant, and all the data would suggest that edaravone does not contribute to ICH [28]. 
Interestingly, in the Enomoto study, it was found that edaravone may potentially reduce the likelihood of ICH [29]. Haemorrhage occurred in $1.4 \%$ and $2.7 \%$ of the experimental and control groups, respectively [29].

\section{Renal Function}

An additional adverse side effect of edaravone administration is renal toxicity $[16,30,31]$. The renal toxicity is thought to stem from increased blood urea and nitrogen concentrations from edaravone, but the direct mechanism is still unknown [26]. In a study of 207 patients treated with edaravone, 17 patients $(8.2 \%)$ were able to attribute their renal disorders to edaravone and 190 patients (91.8\%) had some other associated factor(s) [30]. Out of these patients, $45 \%$ had reversible renal function after edaravone treatment was stopped [30].

\section{Clinical Efficacy}

\section{Functional outcome}

Recently there have been multiple clinical trials observing edaravone impact on functional outcome. One of those was a randomized, multi-centre, placebo-controlled trial of edaravone for 250 acute ischemic stroke patients presenting within 72 hours in 16 sites in Japan [32]. An intravenous dosing schedule of $30 \mathrm{mg}$ twice daily was performed for 14 days [32]. The results demonstrated improved neurological outcomes ( $\mathrm{mRS} \leq 2$ within 90 days) after the administration of edaravone within 72 hours of AIS. This study resulted in the widespread adoption of edaravone for the treatment of AIS patients in Japan [32]. The beneficial effects were most pronounced within 24 hours [16].

A small randomized study conducted in India evaluated 50 patients presenting within 6-72 hours of last known well (LKW) [33]. Patients received $30 \mathrm{mg}$ IV edaravone twice daily for 14 days and improved functional outcome was measured by mRS $\leq 2$ at 90 days [33]. According to the investigators, a statistically significant difference for functional outcome was observed at 90 days (72\%-experimental; $n=25$ vs 42\%-control; $n=25$ ) [33].

The most recent clinical trial, the Enomoto trial, conducted in Japan, included the analysis of 11,508 acute ischemic stroke patients undergoing emergent thrombectomy who received either edaravone or a placebo within 24 hours of LWK [29]. Patients received $30 \mathrm{mg}$ IV edaravone once daily for 14 days, and improved functional outcome was measured at 90 days with $m R S \leq 2$. The stroke severity of patients was measured on the Japan Coma Scale (JCS) where at least $47 \%$ of the edaravone patients had moderately impaired consciousness upon admission. These results also demonstrated statistically significant improved neurological outcome in 32.3\% $(n=10,281)$ and $25.9 \%(n=1,227)$ of the experimental and control groups, respectively [29].

Additionally, in a randomized study involving 17 hospitals in Japan with patients presenting within 4.5 hours of occlusions of the M1 or M2 middle cerebral arteries, the timing of edaravone administration whether before, during or after IV tPA was not significant and neurological improvement occurred at all time frames [28]. This study determined that $60 \mathrm{mg}$ edaravone for 14 days was the optimal dosing regimen to improve functional outcome [28, 33,34].

\section{Improved efficacy of thrombolysis}

Currently tissue plasminogen activator (tPA) is the only FDA approved drug treatment for one of the leading causes of death globally, acute ischemic stroke. While tPA has been found to be a great thrombolytic agent, a vast majority of people miss the beneficial therapeutic time window of IV tPA [6]. The vasodilatory properties of edaravone was found to increase the efficacy of IV tPA $[6,17,26,27,35,36,37]$. This effect was apparent in both large and small-vessel occlusions and it did not matter whether edaravone was administered prior/concurrent with tPA or following tPA infusion $[17,28,38,39]$.

\section{Cerebral edema}

One of the secondary processes that can cause increased infarct growth and neuronal death is cerebral edema. Cerebral edema leads to increased intracranial pressure which may decrease the cerebral perfusion pressure resulting in further ischemia depending on autoregulation [40]. This cerebral edema can be imaged by diffusion MRI and quantified by the amount of restriction showed [41]. Interestingly, it has been found that edaravone attenuates poststroke cerebral edema as well through the regulation of aquaporins [16]. Aquaporin-4 and aquaporin-9 were shown in mice to be the major regulators of water homeostasis in the brain and when acted upon by edaravone have led to reduced cerebral edema [40]. This displays edaravone's multi-functional capabilities at combating ischemic stroke through a variety of biological pathways.

\section{Future Directions}

In order to establish a true connection of edaravone's effects to post-ischemic stroke, a much larger and more diverse population should be studied. The vast majority of patients studied were Japanese and while these data sets are compelling, it is important to confirm this in a more diverse population. To our knowledge, none of the studies included the effects of edaravone on posterior circulation strokes. This is an important patient population to evaluate where functional outcomes and cerebral edema reduction have the potential to have an even greater impact since the deficits are usually greater in these patients. Perfusion imaging is routinely performed in the triage of acute stroke patients and is used to select the high-risk cohort patients who could benefit from endovascular treatment. Stratification of the efficacy of edaravone by perfusion pattern (no/small, medium, and large penumbra) could refine the cohort benefiting from edaravone as well as its magnitude.

While some of the mechanisms of edaravone's efficacy have been studied in animal models, these mechanisms could be confirmed in humans. Other AIS treatments and combination drug treatments, beyond tPA, have also started to be explored. Borneol, a common anti-inflammatory has been studied through clinical patients with AIS. Borneol is thought to help maintain the blood-brain barrier (BBB) and protect against neurodegenerative processes 
[42]. Through 15 studies involving 308 animals, by decreasing the permeability of the BBB, inhibiting inflammation and reducing ROS species, Borneol along with Edaravone helps reduce infarct volume and improve outcome after cerebral infarction [42]. Also through a double blind animal trial it was found Borneol is safe at numerous doses, which makes it a possible drug combination therapy treatment for patients with AIS [43]. "Cocktail” treatments such as this have the potential to synergistically enhance efficacy.

\section{Acknowledgement}

None.

\section{Conflicts of Interest}

The author has no conflicts of interest regarding this paper.

\section{References}

1. (2017) Stroke Facts. Centers for Disease Control and Prevention.

2. (2018) The top 10 causes of death globally. World Health Organization.

3. Uttara B, Singh AV, Zamboni P, Mahajan RT (2009) Oxidative stress and neurodegenerative diseases: a review of upstream and downstream antioxidant therapeutic options. Curr Neuropharmacol 7(1): 65-74.

4. Wheeler HM, Mlynash M, Inoue M, Tipirnini A, Liggins J, et al. (2015) The growth rate of early DWI lesions is highly variable and associated with penumbral salvage and clinical outcomes following endovascular reperfusion. Int J Stroke10(5): 723-729.

5. Bang O, Goyal M, Liebeskind D (2015) Collateral Circulation in Ischemic Stroke. Stroke 46: 3302-3309.

6. Kikuchi K, Kawahara K, Miyagi N, Uchikado H, Kuramoto T, et al. (2010) Edaravone: A new therapeutic approach for the treatment of acute stroke. Med Hypotheses 75(6): 583-585.

7. Filho J, Samuels O (2019) Mechanical thrombectomy for acute ischemic stroke. Up to Date.

8. English JD, Yavagal DR, Gupta R, Janardhan V, Zaidat 00, et al. (2016) Mechanical Thrombectomy-Ready Comprehensive Stroke Center Requirements and Endovascular Stroke Systems of Care: Recommendations from the Endovascular Stroke Standards Committee of the Society of Vascular and Interventional Neurology (SVIN). Interv Neurol 4(3-4): 138-150.

9. Singh, Balwinder Prokop LJ, Mittal MK (2013) Endovascular Therapy for Acute Ischemic Stroke: A Systematic Review and Meta-analysis. Mayo Clin Proc 88(10): 1056-1065.

10. Saver, Jeffrey L (2006) Time is brain-quantified. Stroke 37(1): 263-266.

11. Al-Mufti F, Amuluru K, Roth W, Nuoman R, El-Ghanem M, et al. (2018) Cerebral Ischemic Reperfusion Injury Following Recanalization of Large Vessel Occlusions. Neurosurgery 82(6): 781-789.

12. L L, X W, Z Y (2016) Ischemia-reperfusion Injury in the Brain: Mechanisms and Potential Therapeutic Strategies. Biochem Pharmacol (Los Angel) 5(4): 213.

13. Patel RAG, McMullen PW (2017) Neuroprotection in the Treatment of Acute Ischemic Stroke. Prog Cardiovasc Dis 59(6): 542-548.

14. Ming-Shuo Sun, Hang Jin, Xin Sun, Shuo Huang, Fu-Liang Zhang, et al. (2018) Free Radical Damage in Ischemia-Reperfusion Injury: An Obstacle in Acute Ischemic Stroke after Revascularization Therapy. Oxidative Medicine and Cellular Longevity, pp. 17.

15. Savitz S, Baron J, Sanossian N, Yenari MA, Fisher M (2017) Reconsidering Neuroprotection in the Reperfusion Era. Stroke; 48(12): 3413-3419.

16. Lapchak PA (2010) A critical assessment of edaravone acute ischemic stroke efficacy trials: is edaravone an effective neuroprotective therapy? Expert Opin Pharmacother 11(10): 1753-1763.
17. Kikuchi Kiyoshi, Ko-ichi Kawahara, Tomoka Nagasato, Takuto Terashi, et al. (2017) Edaravone, a Synthetic Free Radical Scavenger, Enhances Alteplase-Mediated Thrombolysis. Oxidative medicine and cellular longevity. pp.14.

18. Watanabe T, Tahara M, Todo S (2008) The Novel Antioxidant Edaravone: From Bench to Bedside. Cardiovasc Ther 26(2): 101-114.

19. Dhanasekaran DN, Reddy EP (2008) JNK signaling in apoptosis. Oncogene 27(48): 6245-6251.

20. Koji Abe, Massahi Aoki, Shoji Tsuji (2017) Safety and efficacy of edaravone in well-defined patients with amyotrophic lateral sclerosis: a randomised, double-blind, placebo-controlled trial. The Lancet.

21. Park HJ, Lee HJ, Choi MS, Son DJ, Song HS, et al. (2008) JNK pathway is involved in the inhibition of inflammatory target gene expression and NF-kappaB activation by melittin. J Inflamm (Lond) 5: 7.

22. Mao YF, Yan N, Xu H, Sun JH, Xiong YC, et al. (2009) Edaravone, a free radical scavenger, is effective on neuropathic pain in rats. Brain Res 1248: 68-75.

23. Yamawaki M, Sasaki N, Shimoyama M, Miake J, Ogino K, et al. (2004) Protective effect of edaravone against hypoxia-reoxygenation injury in rabbit cardiomyocytes. Br J Pharmacol 142(3): 618-626.

24. Tsuruoka A, Atsumi C, Mizukami H, Imai T, Hagiwara Y, et al. (2014) Effects of Edaravone, a Free Radical Scavenger, on Circulating Levels of MMP-9 and Hemorrhagic Transformation in Patients with Intravenous Thrombolysis Using Low-dose Alteplase. J Stroke Cerebrovasc Dis 23(10): 2894-2899.

25. Miller DJ, Simpson JR, Silver B (2011) Safety of thrombolysis in acute ischemic stroke: a review of complications, risk factors, and newer technologies. Neurohospitalist 1(3): 138-147.

26. Moncada S, Vane JR (1979) The role of prostacyclin in vascular tissue. Fed Proc 38(1): 66-71.

27. Li GY, Feng Y, Cheng TS, Yin JM, Zhang CQ (2013) Edaravone, a novel free radical scavenger, prevents steroid-induced osteonecrosis in rabbits. Rheumatology 52(3): 438-447.

28. Aoki J, Kimura K, Morita N, Harada M, Metoki N, et al. (2017) YAMATO Study (Tissue-Type Plasminogen Activator and Edaravone Combination Therapy). Stroke 48(3): 712-719.

29. Enomoto M, Endo A, Yatsushige H, Fushimi K, Otomo Y (2019) Clinical effects of early Edaravone use in acute ischemic stroke patients treated by endovascular reperfusion therapy. Stroke 50(3): 652-658.

30. Hishida A, (2007) Clinical analysis of 207 patients who developed renal disorders during or after treatment with edaravone reported during post-marketing surveillance. Clin Exp Nephrol 11(4): 292-296.

31. Hishida A (2009) Determinants for the prognosis of acute renal disorders that developed during or after treatment with edaravone. Clin Exp Nephrol 13(2): 118-122.

32. Edaravone Acute Infarction Study Group (2003) Effect of a novel free radical scavenger, edaravone (MCI-186), on acute brain infarction. Randomized, placebo-controlled, double-blind study at multi centers. Cerebrovasc Dis 15(3): 222-229.

33. Sinha M, Shukla R, Garg RK, Verma R, Singh MK, et al. (2011) A randomized controlled clinical trial to compare the safety and efficacy of edaravone in acute ischemic stroke. Ann Indian Acad Neurol 14(2): 103-106.

34. Feng S, Yang Q, Liu M, Li W, Yuan W, et al. (2011) Edaravone for acute ischaemic stroke. Cochrane Database Syst Rev (12): CD007230.

35. Sun YY, Morozov VM, Yang D, Li Y, Dunn RS, et al. (2014) Synergy of Combined tPA-Edaravone Therapy in Experimental Thrombotic Stroke. PLoS ONE 9(6): e98807.

36. Miyaji Y, Yoshimura S, Sakai N, Yamagami H, Egashira Y, et al. (2015) Effect of Edaravone on Favorable Outcome in Patients with Acute Cerebral Large Vessel Occlusion: Subanalysis of RESCUE-Japan Registry. Neurol Med Chir (Tokyo) 55(3): 241-247. 
37. Takenaka K, Kato M, Yamauti K, Hayashi K (2014) Simultaneous Administration of Recombinant Tissue Plasminogen Activator and Edaravone in Acute Cerebral Ischemic Stroke Patients. J Stroke Cerebrovasc Dis 23(10): 2748-2752.

38. Nakase T, Yoshioka S, Suzuk A (2011) Free radical scavenger, edaravone, reduces the lesion size of lacunar infarction in human brain ischemic stroke. BMC Neurol 11: 39

39. Toyodaa K, Fujiib K, Kamouchia M, Nakane H, Arihiro S, et al. (2004) Free radical scavenger, edaravone, in stroke with internal carotid artery occlusion. J Neurol Sci 221(1-2): 11-17.

40. Badaut J, Lasbennes F, Magistretti PJ, Regli L (2002) Aquaporins in brain: distribution, physiology, and pathophysiology. J Cereb Blood Flow Metab 22(4): 367-378.
41. Koch S, Rabinstein A, Falcone S, Forteza A (2001) Diffusion-weighted Imaging Shows Cytotoxic and Vasogenic Edema in Eclampsia. AJNR Am J Neuroradiol 22(6): 1068-1070.

42. Chen ZX, Xu QQ, Shan CS, Shi YH, Wang Y, et al. (2019) Borneol for Regulating the Permeability of the Blood-Brain Barrier in Experimental Ischemic Stroke: Preclinical Evidence and Possible Mechanism. Oxid Med Cell Longev: 936737.

43. Xu J, Wang Y, Wang A, (2019) Safety and efficacy of Edaravone Dexborneol versus edaravone for patients with acute ischaemic stroke: a phase II, multi centre, randomised, double-blind, multiple-dose, active-controlled clinical trial. Stroke and Vascular Neurology: 000221. 